


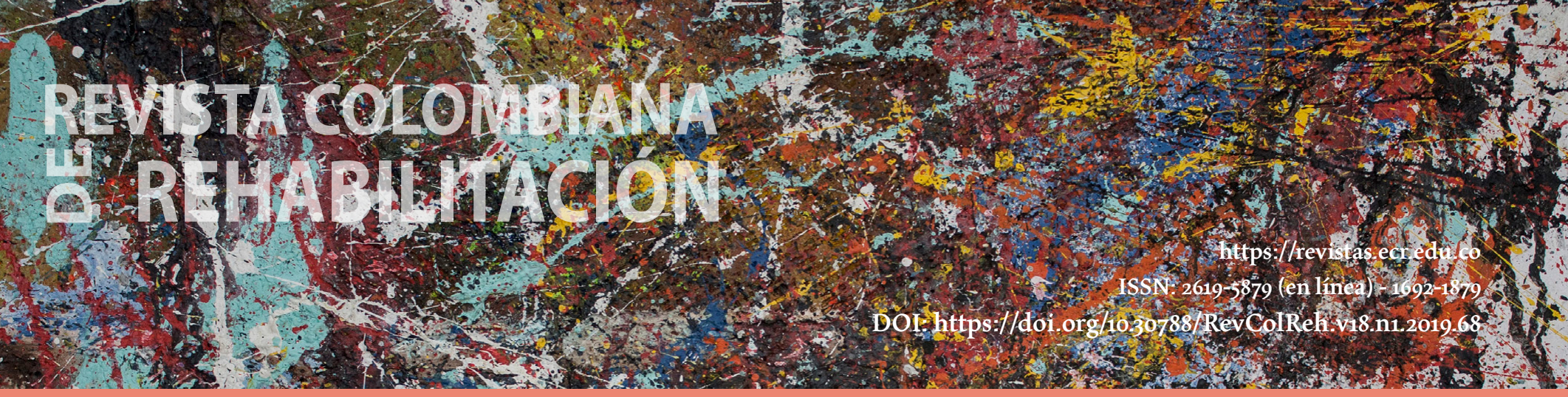

Fecha de recepción: 08 de septiembre de 2017

Fecha de aprobación: 10 de mayo de 2018

\title{
Influencia de un programa de estimulación temprana en el desarrollo lingüístico de niños de Sincelejo
}

Madelin María Palacio Vásquez
https://orcid.org/oooo-0003-4260-7540

Marta Lucía Hernández Blanco ${ }^{a}$
Yaniris del Carmen Álvarez Pérez ${ }^{\mathrm{a}}$

Pedro José Blanco Tuirán ${ }^{\mathrm{a}}$
Ana María Gómez Benítez ${ }^{\mathrm{a}}$

Justo Rafael Fuentes Cuello ${ }^{a}$

'Universidad de Sucre, Sincelejo, Colombia

\section{Resumen}

Existe evidencia de los beneficios de la estimulación temprana sobre el desarrollo de capacidades y habilidades de niños en condición de riesgo biológico o social, también se conoce sobre el impacto negativo a la falta de estimulación sobre el desarrollo. Objetivo: determinar el impacto de un programa de estimulación temprana en el desarrollo del lenguaje en niños de 3 años del municipio de Sincelejo. Método: Participaron 58 niños de 3 a 3,5 años valorados y divididos en dos grupos diagnósticos de retraso y normalidad en el desarrollo, divididos a su vez en grupos de intervención y controles. Las valoraciones de desarrollo del lenguaje inicial, intermedias y final se realizaron con PLON-R. El programa de intervención tuvo una duración de 8 meses y se basó en la estimulación del lenguaje oral en niños de 3 años. Se realizaron análisis mediante la prueba estadística de Wilcoxon de los rangos con signo, se estableció la significancia estadística. Resultados: se demostró que el programa de estimulación diseñado y ejecutado en este estudio fue efectivo, permitiendo que los niños mejoraran sus habilidades de lenguaje. Discusión: Se confirman los hallazgos previos en torno a desarrollo del lenguaje y el papel de la estimulación en los logros, tanto lingüísticos como cognitivos.

Palabras clave: Lenguaje infantil; terapia del lenguaje, desarrollo del lenguaje; trastornos del desarrollo del lenguaje.

\section{Influence of early learning stimulation program in linguistic development of children in Sincelejo}

\begin{abstract}
There is evidence of the benefits of early stimulation on the development of abilities and abilities of children in biological or social risk condition, it is also known about the negative impact to the lack of stimulation on development. Objective: to determine the impact of an early stimulation program on language development in 3-year-old children in the municipality of Sincelejo. Method: 58 children aged 3 to 3.5 years assessed and divided into two diagnostic groups of delay and normality in development, divided in turn into intervention and control groups. The initial, intermediate and final language development evaluations were carried out with PLON-R. The intervention program lasted 8 months and was based on the stimulation of oral language in 3-year-old children. Analyzes were carried out using the Wilcoxon statistical test of signed ranges, statistical significance was established. Results: it was demonstrated that the stimulation program designed and executed in this study was effective, allowing the children to improve their language skills. Discussion: Previous findings regarding language development and the role of stimulation in linguistic and cognitive achievements are confirmed.
\end{abstract}

Key words: Child language; language therapy; language development; language development disorders. 


\section{Introducción}

La estimulación temprana es un grupo de técnicas educativas especiales empleadas entre el nacimiento y los seis años de vida, con el fin de posibilitar la adopción de medidas encaminadas a la prevención, la detección precoz de riesgos a deficiencias y la intervención de los diversos trastornos del desarrollo, lo cual aplica para niños sanos (estimulación y potencialización de habilidades), que tienen o están en riesgo de adquirir un retraso en el desarrollo (Majnemer, 1998) y patológicos (intervención de trastornos). Por ende, la implementación de programas de estimulación de lenguaje en este período de vida es primordial, por ser la etapa base para el desarrollo de las capacidades cognitivas, emocionales, físicas y mentales en los niños, permitiéndoles adquirir de forma eficaz otras habilidades, para mejorar su calidad de vida (Secretaría de Salud de México, s.f).

Según Azcoaga et al. (1992), el lenguaje es una función cerebral superior que se adquiere mediante un proceso de aprendizaje que cursa gracias a la existencia de suficientes estímulos verbales en el medio. Por tal razón, requiere de procesos de estimulación para su adecuada adquisición en todas sus dimensiones: forma, contenido y uso.

La forma del lenguaje engloba los aspectos fonológicos, fonéticos y morfosintácticos. La fonética analiza los sonidos (realizaciones físicas); mientras que la fonología analiza los fonemas, que son abstracciones, estudia su forma y organización (Saussure, 1973). La morfo-sintaxis cumple la función de combinar las piezas léxicas de una lengua, con el fin de formar y estructurar oraciones (Hernanz \& Brucart, 1987). La semántica es lo que representa la dimensión del contenido, y se encarga de estudiar el significado de las palabras de una lengua (Gallardo \& Gallego, 1995). La Pragmática es concebida como el uso del lenguaje en función del contexto que le concierne, respeto de turnos de intervención, modificación del discurso en función del interlocutor, aplicación de funciones del lenguaje etc. (Miretti, 2003).

Dentro de las dificultades del lenguaje en la edad temprana, se destaca el retraso simple del lenguaje (RSL), que consiste en la aparición más tardía de lo habitual y un desarrollo lingüístico lento y desfasado con respecto a lo esperado para la edad cronológica del niño. Esta disfunción no tiene relación con la capacidad intelectual, ni con alteraciones motrices o sensoriales (Serna \& Gil, 2014). Los niños con RSL muestran patrones fonológicos desviados, su habla suele ser infantilizada y la estructura silábica es simple. Un elemento clave a trabajar en los aspectos fonético-fonológicos es la conciencia fonológica, especialmente en lenguas fonológicamente transparentes como es el castellano (De la Torre, Guerrero, Conde \& Claros, 2002). El componente morfosintáctico de infantes con RSL se caracteriza por desorganización en la sucesión normal de la oración (el lenguaje presenta un aspecto telegráfico). El número de conceptos que nombran en la frase es reducido, y tienen dificultades en el manejo de artículos, pronombres, plurales y en el uso de diferentes tiempos verbales (Peña, 2014). En la dimensión contenido del lenguaje (aspectos semánticos) el vocabulario está normalmente mermado, basándose en objetos del entorno más cercano, presentan dificultades para la asimilación de conceptos abstractos, como colores, formas y tamaños o nociones espacio temporales (Alegre \& Pérez, 2008). En la Pragmática se evidencian falencias en la intención comunicativa, pues el lenguaje es utilizado básicamente para nombrar y conseguir objetos, así como para estructurar la conducta. Los niños con RSL muestran dificultades para explicar las cualidades de objetos y articular preguntas; apoyan sus exposiciones verbales con excesiva mímica y gestos para compensar las carencias expresivas. La terapia en esta dimensión se enfoca en trabajar las funciones del lenguaje y habilidades conversacionales (Serna \& Gil, 2014).

Muchos estudios se han encargado de explicar ampliamente los beneficios de la estimulación temprana para el desarrollo de las capacidades y habilidades de los niños y niñas en condición de riesgo biológico o social, así como también han demostrado el impacto negativo a la falta de estimulación para el desarrollo integral del niño (Reyes Cuervo \& Bayona González, 2010).

El origen de los programas de estimulación temprana nació en Sudamérica en los países de Uruguay y Chile. Las actividades se iniciaron en Uruguay en 1963, con trabajos exclusivamente con niños que presentaban retardo mental y a partir de 1967 se comenzó a utilizar la estimulación precoz con niños de alto riesgo (Equipo Facemamá, 2015). Posteriormente se fueron desarrollando diversos programas en Chile, Venezuela y otros países, cuyos estudios más recientes se mencionan a continuación: 
En la Ciudad de Loja y Graciela Aterihuana de Cueva (Ecuador) en los años 2009-2010, se realizó un estudio, cuyo objetivo fue investigar la incidencia de la estimulación temprana en el desarrollo del lenguaje oral de los niños y niñas de los centros de educación inicial de esta ciudad. La población estuvo conformada por niños entre los o y 6 años de edad. Los métodos y técnicas utilizados en esta investigación fueron el científico, descriptivo y estadístico, empleados con el fin de describir y explicar, la forma y la relación que guardan la estimulación temprana y el desarrollo del lenguaje oral de los infantes. El instrumento empleado fue una encuesta aplicada a las maestras y padres de familia para conocer la estimulación temprana que reciben los niños; una prueba de funciones básicas para conocer el desarrollo del lenguaje oral. Realizados estos procesos se analizó y contrastó la información empírica y el marco teórico, y se concluyó que la estimulación temprana influye en el desarrollo del lenguaje oral, por lo cual se puede afirmar que existe una relación significativa entre las variables, es decir que a una mejor estimulación temprana demuestran un mejor desarrollo del lenguaje oral (Lalangui, Romero \& Escudero, 2014).

Por otra parte, se realizó un estudio en 2004 en el estado de Michoacán México por un periodo de 40 semanas con una población de 7.229 niños: 4.174 experimentales y 3.055 controles en niños menores de cuatro años pertenecientes a familias deprimidas, en el cual se utilizó para la valoración del desarrollo la "Escala Jalisco", antes y después de la estimulación. Antes de iniciar el estudio se capacitó y entrenó a los padres en estimulación temprana. Los resultados señalaron que antes de iniciar la "estimulación temprana" el desarrollo del grupo experimental era superior al del grupo control, pero la diferencia fue aun de mayor significación al concluir el estudio (Pando, Aranda, Amezcua, Salazar \& Torres, 2004).

En Colombia se están implementando programas de estimulación en niños de o a 6 años de edad, en grandes ciudades como Bogotá y Medellín, realizándose dos estudios: El primero, denominado: "Desarrollo infantil temprano y brecha socio-económica", midió la brecha del desempeño cognitivo y no-cognitivo de niños en Bogotá entre 6 y 42 meses, los resultados mostraron que existen brechas significativas en desarrollo cognitivo y de lenguaje a partir de los 17 y 9 meses de edad, respectivamente, entre los niños pertenecientes a hogares de estrato 1 y los de estrato 3 y 4 . Esta brecha incrementa en un $57 \%$ en desarrollo cognitivo y en un $83 \%$ en los infantes entre los 18 y los 42 meses (Maldonado \& Oliva, 2008). El segundo, realizó una evaluación de impacto de una intervención piloto en estimulación infantil temprana y nutrición en 96 municipios semi-rurales del Programa Familias en Acción en los departamentos de Cundinamarca, Boyacá y Santander; Antioquia, Risaralda y Caldas; Huila y Tolima. El estudio encontró impactos importantes en el desarrollo cognitivo, de lenguaje, y sobre la cantidad de tiempo y dinero que la familia invierte en sus hijos. (Attanasio \& Gómez, 2012).

A nivel regional, local e institucional, solo se reportan los resultados de una pasantía realizada en el municipio de Corozal- Sucre en la ESE (Entidad de Salud del Estado) Cartagena de Indias, durante el segundo periodo de 2009, donde se instauró un programa de actividades asistenciales durante cuatro (4) meses, encaminado a la intervención individual de pacientes con desórdenes comunicativos en el área del lenguaje oral. En este tiempo se registró un total de veinte (20) pacientes, con edades que oscilaban entre los cuatro (4) y once (11) años a los cuales, aplicaron pruebas estandarizadas (el test de CELF, Teprosif y Terman) y no estandarizadas. Las valoraciones realizadas permitieron establecer que un $60 \%$ de la población tuvo problemas en el desarrollo pragmático y semántico, mientras que el 40\% restante mostró dificultades en los procesos fonético-fonológicos y sintácticos. En los niños de cuatro a cinco años, que correspondían al 35\% ( $\mathrm{n}=7$ ), según las dificultades reportadas se les emitió el diagnóstico de retardo simple del lenguaje. Estos, fueron sometidos a un proceso de intervención y, mostraron avances significativos en su lenguaje oral; sin embargo, dos de ellos (10\%), no lograron superar en su totalidad las dificultades. Es de resaltar que estos fueron aquellos que presentaron problemas tanto a nivel fonético como morfosintáctico (Petano, 2009).

Ante esta dificultad lingüística de la edad temprana, lo más aconsejable es estimular al niño para que su aprendizaje verbal sea típico, pero si no fuera así, es preciso recurrir a un fonoaudiólogo para ayudarle en su aprendizaje (González, 2013). Debido a esto, el objetivo principal del presente estudio experimental fue determinar el impacto de un programa de estimulación temprana en el desarrollo del lenguaje en niños de 3 años de la ciudad colombiana de Sincelejo. Teniendo en cuenta que, en las edades de 3, 6 y 11 años hay un gran despegue en el desarrollo del lenguaje y que en la etapa de los 3 años la plasticidad del cerebro aún está en proceso (Del Rio, 1987 citado en Brodal, 2010; Luciana, 2003). 


\section{Método}

La investigación se desarrolló con un enfoque cuantitativo, con un estudio causal de diseño cuasi-experimental, los cuales son un tipo de investigación concluyente que tiene como prioridad obtener evidencia de la relación causa y efecto de un fenómeno. Los estudios experimentales permiten manipular intencionalmente una o más variables independientes (supuestas causas-antecedentes), para analizar las consecuencias que la manipulación tiene sobre una o más variables independientes (supuestos efectos-consecuentes), dentro de una situación de control para el investigador, es decir, manipulan tratamientos, influencias o intervenciones (variables independientes) para observar sus efectos sobre otras variables (dependientes) (Hernández, Fernández \& Baptista, 2014).

Para este estudio se valoró el estado inicial del desarrollo del lenguaje y luego se determinó la influencia del programa de estimulación temprana diseñado y ejecutado en el desarrollo lingüístico de los niños de 3 años.

\section{Participantes}

Los sujetos seleccionados en la investigación correspondieron a todos los niños de 3 años, adscritos a dos Centros de Desarrollo Infantil (CDI) de la zona sur y un Hogar Infantil ubicado al oriente; ambos en el municipio de Sincelejo, departamento de Sucre. La muestra estuvo conformada por 58 niños en edades comprendidas entre los 3.0 y 3.5 años de edad seleccionados mediante un muestreo aleatorio simple por medio del método de selección coordinado-negativo y que cumplieron con los criterios de inclusión: normalidad o retraso simple del lenguaje. Se excluyeron los niños que presentaban disminución auditiva, media nte la cartilla de instrumentos de tamizaje para la detección temprana de deficiencias auditivas en los menores de cinco años y escolares del INSOR (INSOR, 2009); infantes con discapacidad cognitiva, signos de alteración neurológica, trastorno específico del lenguaje o alteraciones en órganos fono-articuladores verificados, a través de la realización de una anamnesis a los padres de familia y la evaluación del lenguaje.

\section{Instrumentos}

La técnica utilizada fue evaluativa, a través de la Prueba de Lenguaje Oral de Navarra Revisada PLON-R de Aguinaga, Armentia, Fraile, Olangua y Uriz (2005), la cual evalúa el desarrollo del lenguaje oral de los niños de 3, 4, 5 y 6 años en los aspectos de Forma, Contenido y Uso del lenguaje para realizar una detección rápida o tamiz del desarrollo lingüístico. De acuerdo a la edad del niño se evalúa cada uno de los aspectos del lenguaje con las actividades propuestas en la prueba siguiendo las instrucciones dadas en cada actividad.

La calificación se realiza como plantea el formato de aplicación y manual de la prueba, luego se suman los puntajes por componente del lenguaje y también de forma general, la puntuación obtenida se compara con las tablas de Baremos propuestas en la prueba. Esto da como resultado tres diagnósticos o categorías: 1) Normal (N): puntuación media o por encima de la media; 2) Necesita Mejorar (NM): puntuación entre la media y una desviación típica por debajo de la media y 3) Retraso (R): puntuación inferior a una desviación típica por debajo de la media.

Los datos obtenidos en los diferentes Centros de Desarrollo Infantil (CDI) se registraron en los formularios de cada uno de los formatos de anamnesis y pruebas aplicadas. Posteriormente, fueron introducidos los resultados, en los programas Epilnfo 7 y R (Team, 2015). En el primero se codificó y digitó la encuesta aplicada; y se utilizaron ambos programas para depurar los datos. A su vez, estos se procesaron y analizaron mediante tablas de distribución de frecuencias, análisis exploratorio y de contingencia. El análisis de comparación antes y después de los grupos y las distintas evaluaciones y revaloraciones se realizó por medio de la Prueba de Wilcoxon de los rangos con signo.

\section{Procedimiento}

Para llevar a cabo el estudio se consideraron 6 fases: En la primera se les solicitó a los padres de familia diligenciar y firmar el consentimiento informado, luego se recolectaron datos de anamnesis y se realizó la evaluación del lenguaje y audición. En la segunda se analizaron los datos de la anamnesis y los resultados de las valoraciones y se diagnosticó el desarrollo lingüístico de los niños, como se señaló arriba en las categorías expuestas en el párrafo anterior (teniendo en cuenta los criterios de inclusión y exclusión establecidos). En la tercera se conformaron los diferentes grupos del estudio, de la siguiente forma: Grupo 1: Niños con retardo simple del lenguaje a intervenir; grupo 2: Niños con retardo simple del 
lenguaje del grupo control; grupo 3: Niños con normalidad en el lenguaje a intervenir; grupo 4: Niños con normalidad en el lenguaje del grupo control.

Un cuarto paso fue el diseño del programa de estimulación temprana y su aplicación en la población seleccionada. Este programa se desarrolló durante 8 meses, 2 veces por semana con una duración de 40 minutos por sesión, realizándose en total 64 sesiones de estimulación. En este se desarrollaron diversas actividades, dentro de las cuales se encontraban: actividades de juego, utilización de material didáctico específico para la edad, denominación, imitación diferida, repetición, discriminación auditiva, clasificación, interacción espontánea, juegos de roles, cuentos, rondas infantiles, estrategias encaminadas a favorecer el desarrollo de las diferentes habilidades de los niveles del lenguaje, teniendo en cuenta las dimensiones de forma (morfología y sintaxis), contenido (semántica) y uso (pragmática), tomando como referente no solo un modelo de intervención si no una conjugación de diversos aspectos basados en los modelos lingüístico, conductual y cognitivo, debido a que estos son una guía fundamental, porque una de sus funciones es proporcionar una representación inteligible de una teoría, generar hipótesis susceptibles de comparación y facilitar conocimientos desde el campo de estudio que se aborde una investigación.

En la quinta fase, se realizaron las respectivas revaloraciones ( 3 en total) y se tabularon los resultados arrojados por la prueba PLON-R en cada dimensión del lenguaje, para los 4 grupos del estudio. Finalmente, se analizaron los resultados y se realizaron las respectivas comparaciones antes y después de los grupos de estudios y las revaloraciones, a través de la prueba estadística de Wilcoxon de los rangos con signo, para establecer si existía o no significancia estadística, haciendo también la respectiva descripción de dicho análisis, con el fin de determinar los beneficios y la influencia de la estimulación temprana en el desarrollo lingüístico de los niños, lo que a su vez da respuesta a los interrogantes plasmados en la investigación, de acuerdo a los resultados y estadísticas arrojadas por el estudio.

\section{Resultados}

A continuación, se presenta el análisis descriptivo y estadístico de los resultados arrojados en la ejecución del programa de estimulación del lenguaje:

\section{Análisis descriptivo}

Evaluación inicial del lenguaje. Para la evaluación inicial del lenguaje, se contó con un total de 96 niños. Como se aprecia en la figura 1, 19 (20\%) exhibieron normalidad en el desarrollo lingüístico, 25 (26\%) obtuvieron un perfil de necesitar mejorar su lenguaje (NM), y 52 (54\%) niños se ubicaron con un diagnóstico de retraso en su desarrollo lingüístico, lo cual indica que, más del $50 \%$ de la población presentó retraso.

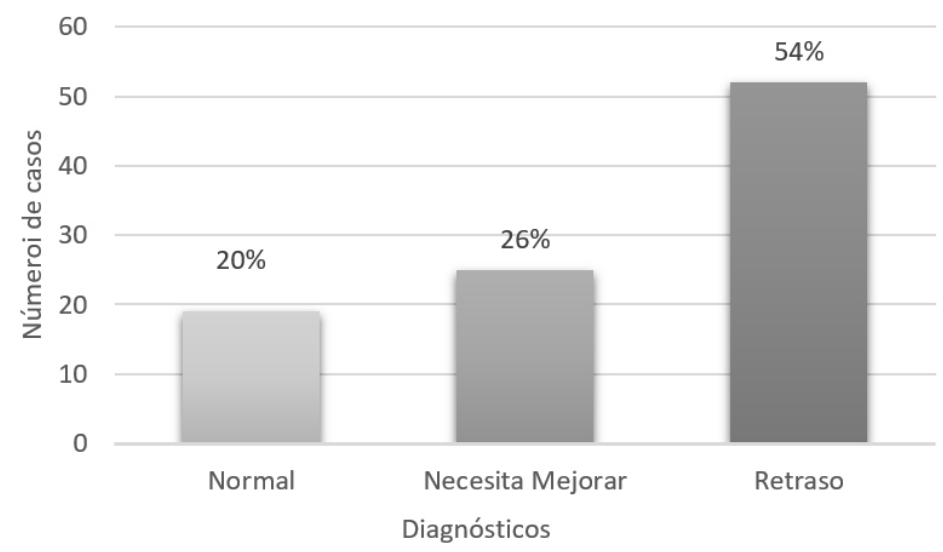

Figura 1. Diagnósticos resultantes de la evaluación inicial del desarrollo lingüístico de los niños y niñas participantes del estudio.

Del total de los niños con retraso simple del lenguaje se excluyeron aquellos que no cumplieron los criterios de inclusión. Por tanto, la muestra para la presente investigación se constituye de 40 niños y niñas con retraso simple del lenguaje 
(RSL), los cuales fueron asignados aleatoriamente y de manera equitativa a dos grupos; un grupo para estimulación del lenguaje (Grupo de intervención) y otro como grupo control. En cuanto a los sujetos con perfil de normalidad o desarrollo normal del lenguaje (DNL), 10 fueron asignados al grupo de intervención y 8 al grupo control. En la figura 2 se observa esta distribución de los participantes en los grupos.

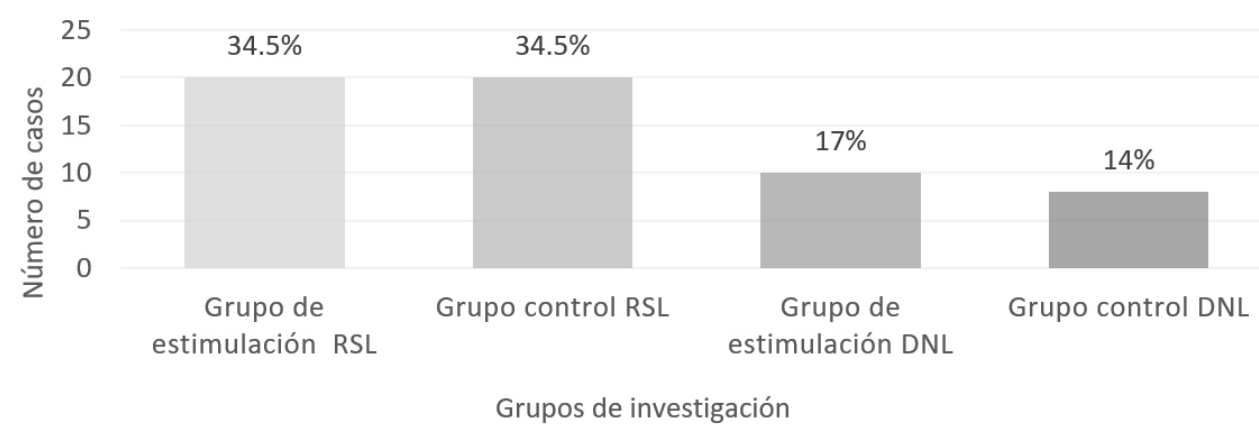

Figura 2. Distribución de los participantes en los grupos de intervención y control de acuerdo con su diagnóstico de desarrollo lingüístico (retraso simple del lenguaje - RSL y desarrollo normal del lenguaje - DNL)

La figura 3 permite evidenciar que hubo disminución de la muestra en cada una de las revaloraciones y grupos del estudio, excepto en el grupo control con desarrollo normal del lenguaje (DNL) en el que se mantuvo el número de participantes en la primera y segunda revaloración, sin embargo, en relación a la evaluación inicial y final disminuyó.

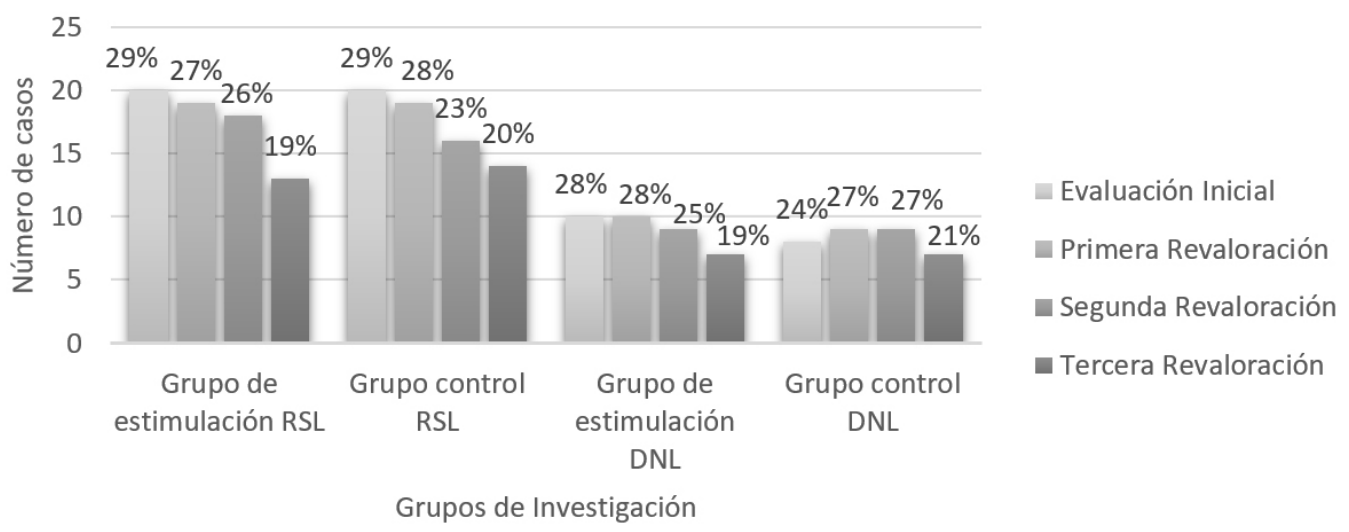

Figura 3. Variaciones en la muestra de los grupos de estimulación y grupos controles

\section{Comparación de la evolución de perfiles generales del lenguaje en los grupos con RSL y DNL según PLON-R}

\section{Primera revaloración}

Grupos con RSL. En el grupo de estimulación, 7(38\%) de los niños revalorados se ubicaron en normalidad ante la prueba, $10(54 \%)$ en la categoría necesita mejorar (NM), mientras que $2(8 \%)$ en retraso. En el grupo control con RSL ninguno de los niños mostró normalidad ante la prueba, 7(37\%) se situaron en Necesita de Mejorar (NM), mientras que el mayor porcentaje (63\%) se encontró en el perfil de retraso pues hubo 12 niños clasificados en este grupo. Como se aprecia en la figura 4 , los niños intervenidos durante el primer trimestre de estimulación tuvieron mejor rendimiento lingüístico que el grupo control.

Grupos con DNL. Los participantes del grupo estimulado con normalidad en el lenguaje potencializaron sus habilidades lingüísticas, obteniendo mayores puntajes en la prueba aplicada (PLON-R), manteniéndose, dentro de los rangos de normalidad. No obstante, 5 niños del grupo control presentaron un descenso en el resultado de la prueba, desviándose de los parámetros de normalidad como se observa en la figura 4. Esto puede explicarse teniendo en cuenta factores 
socioeconómicos, psicoafectivos y falta de estimulación familiar, debido a que los menores pertenecen en su mayoría los estratos 1 y 2, donde es característico la disfuncionalidad familiar, la precariedad en el cubrimiento de necesidades básicas, entre otros aspectos, que obligan a los padres a mantener a sus hijos por largos período de tiempo en los hogares infantiles o CDI.

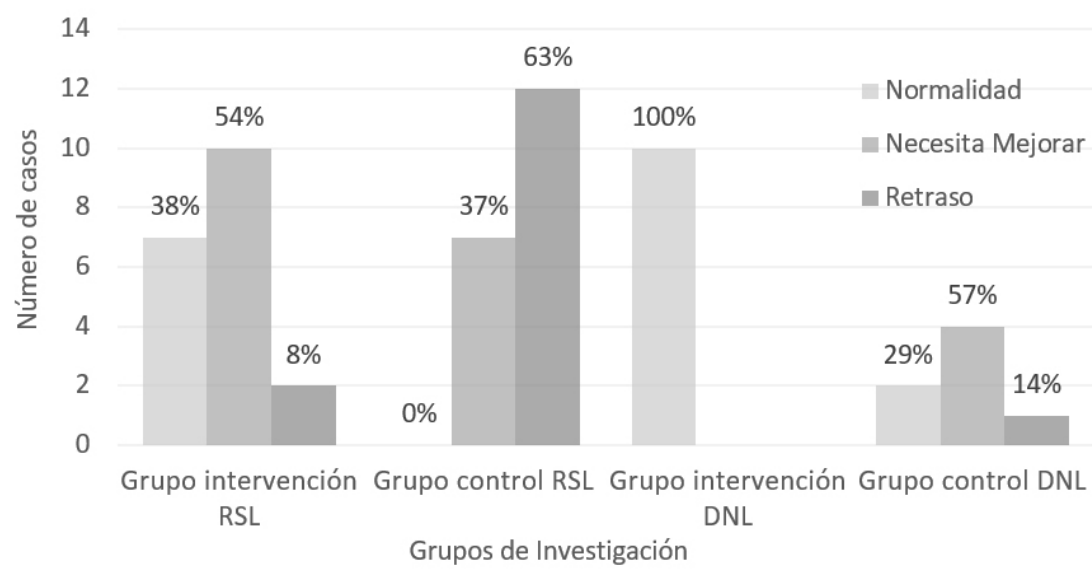

Figura 4. Comparación evolutiva de perfiles generales del lenguaje en los grupos con RSL y DNL según PLON-R en la primera revaloración

\section{Segunda revaloración}

Grupos con RSL. En el grupo de estimulación el 100\% de los niños mostró normalidad (N), lo que, según se observa en la figura 5, muestra una tendencia de aumentar las puntaciones en cada una de las dimensiones del lenguaje con respecto a la prueba. En el grupo control, 2 (12\%) de los niños figuraron en el perfil de normalidad (N), 6(38\%) se ubicaron en la categoría necesita mejorar, mientras que 8 (50\%) clasificaron en el perfil de retraso. Lo anterior evidencia el desempeño lingüístico satisfactorio en los niños intervenidos, aspecto que corrobora la efectividad de los programas de estimulación en la población estudiada

Grupos con DNL. En la segunda revaloración se evidenció que el 100\% de los niños permanecieron en normalidad en ambos grupos. Sin embargo, los puntajes obtenidos en la prueba de los intervenidos son mayores con un rango de 13 a 14 puntos, en comparación al grupo control que evidencia un rango de 11 a 13 puntos. El puntaje máximo de la prueba son 14 puntos.

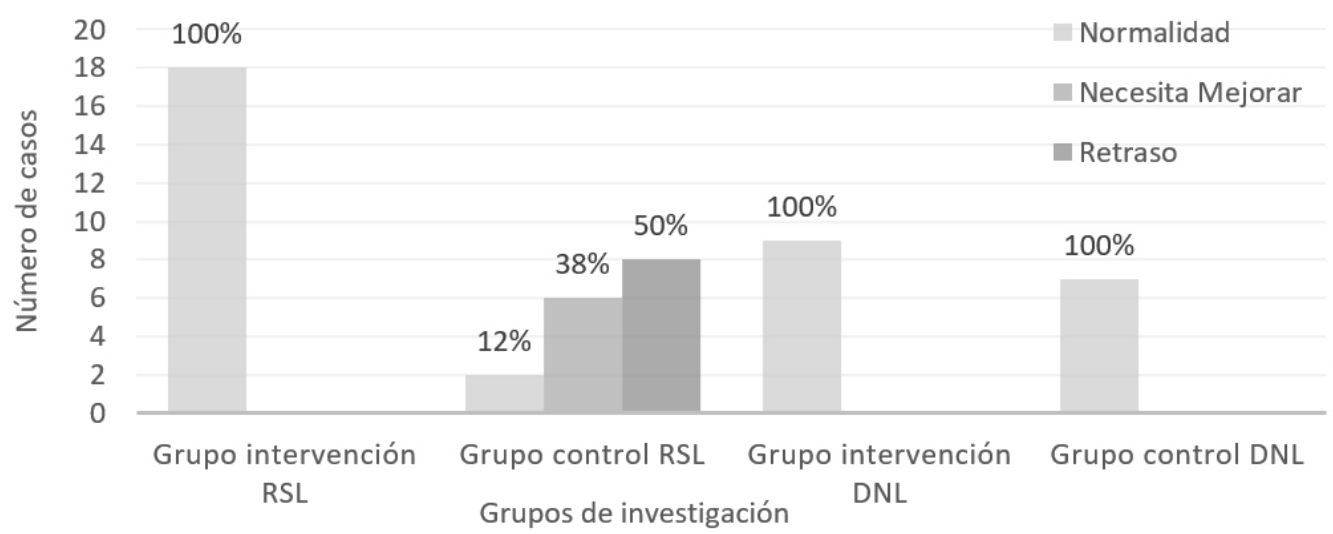

Figura 5. Comparación evolutiva de perfiles generales del lenguaje en los grupos con RSL y DNL según PLON-R en la segunda revaloración 


\section{Tercera revaloración}

Grupos con RSL. Con relación a los resultados en el grupo de estimulación solo una niña presentó retraso del lenguaje, lo cual se atribuye a la edad, debido a que en el momento de aplicar la prueba recién cumplía los cuatro años, por lo que fue sometida a la valoración concerniente a dicha edad, que contiene mayores exigencias en todas las habilidades lingüísticas y estas pueden ser adquiridas a lo largo de los cuatro años. En el grupo control el 14\% alcanzaron el perfil de normalidad, mientras que el 50\% mejoraron sus habilidades del lenguaje (perfil de necesita mejorar) y el $36 \%$ persistieron con retraso.

Grupos con DNL. La evaluación final mostró que el 100\% del grupo estimulado presentaron normalidad. La figura 6 muestra que el grupo control 3 niños permanecieron en el perfil de normalidad, 1 se ubicó en necesita mejorar sus habilidades y 1 en retraso, este último se le atribuye a la edad.

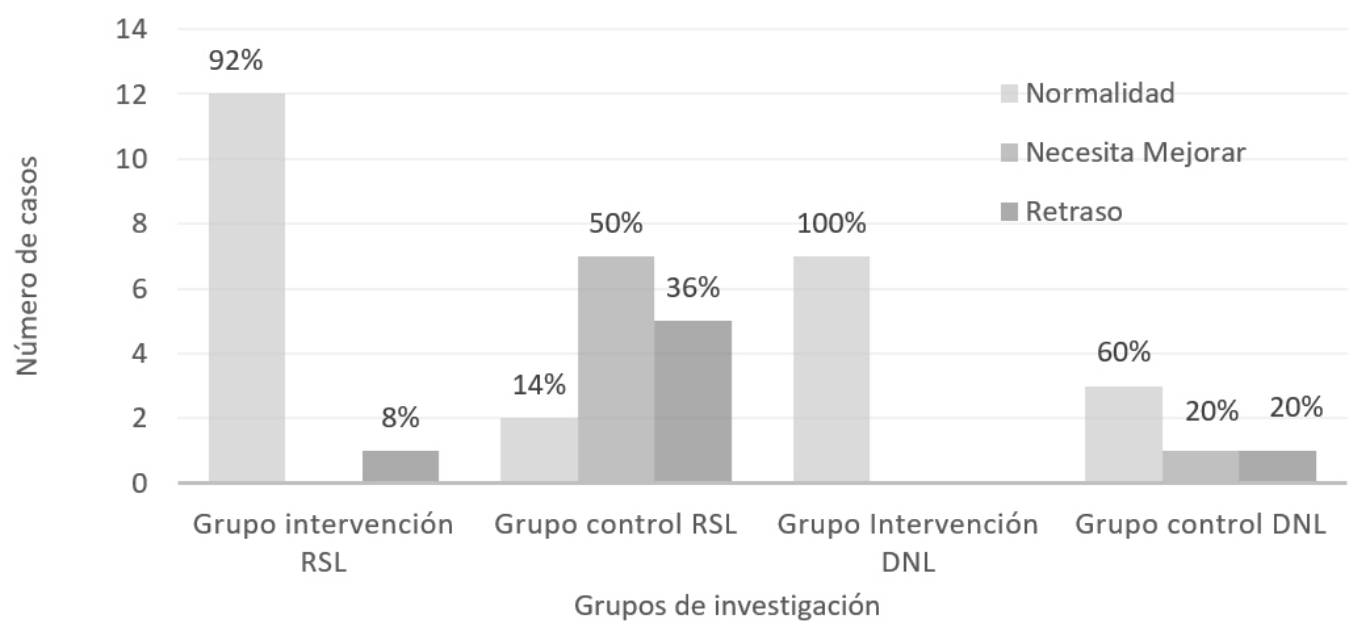

Figura 6. Comparación evolutiva de perfiles generales del lenguaje en los grupos con RSL y DNL según PLON-R en la tercera revaloración.

\section{Análisis estadístico}

Se aplicó la Prueba de Wilcoxon de los rangos con signo, la cual reportó los valores de probabilidad para las siguientes relaciones:

Ho (hipótesis nula): No hay Diferencia (no hay significancia estadística)

Ha (hipótesis alternativa): Sí hay Diferencia (sí hay significancia estadística).

Criterio: Si el valor de la Probabilidad es menor que 0,05 (5\%) entonces se rechaza la Ho.

Comparación de la significancia estadística de las pruebas de Wilcoxon de rangos con signo del grupo intervención y grupo control RSL. Con relación a la primera y segunda revaloración, la Prueba de Wilcoxon de los rangos con signos reportó en los grupos control e intervención significancia estadística para todas las dimensiones del lenguaje y para las puntuaciones totales (exceptuando la dimensión de uso en la primera revaloración en el grupo control). Sin embargo, estas son mayores en los niños intervenidos del grupo con retraso, lo cual indica que estos alcanzaron mejor rendimiento lingüístico que los no estimulados, de los cuales algunos siguen su curso de desarrollo del lenguaje y mejoraron sus habilidades comunicativas, pero en menor proporción que el grupo de intervención, mientras que otros persistían con retraso lingüístico.

Contrario a esto, se observó que en la tercera revaloración la significancia estadística en ambos grupos fue similar, lo cual puede explicarse porque la muestra del estudio disminuyó, debido a la deserción de los niños por periodo de vacaciones, y en el grupo control muchos niños mejoraron sus habilidades de lenguaje, pero sólo 2 de ellos alcanzaron el perfil de normalidad, lo cual varía en gran manera con la valoración inicial donde todos los niños tenían retraso del lenguaje como se observa en la tabla 1 . 
Tabla 1.

Comparación de Significancia estadística de las pruebas de Wilcoxon de rangos con signo del grupo intervención y grupo control RSL.

\begin{tabular}{|c|c|c|c|c|}
\hline \multicolumn{5}{|c|}{ Evaluaciones y revaloraciones } \\
\hline \multicolumn{5}{|c|}{ Relación de la Primera Revaloración y Evaluación Inicial del lenguaje } \\
\hline Grupo & Forma & Contenido & Uso & Total \\
\hline Control & 0,012 & 0,008 & 0,589 & 0,004 \\
\hline Intervención & 0,000 & 0,000 & 0,013 & 0,000 \\
\hline \multicolumn{5}{|c|}{ Relación de la Segunda Revaloración y Evaluación Inicial del lenguaje } \\
\hline Grupo & Forma & Contenido & Uso & Total \\
\hline Control & 0,002 & 0,002 & 0,013 & 0,001 \\
\hline Intervención & 0,000 & 0,000 & 0,000 & 0,000 \\
\hline \multicolumn{5}{|c|}{ Relación de la tercera Revaloración y Evaluación Inicial del lenguaje } \\
\hline Grupo & Forma & Contenido & Uso & Total \\
\hline $\begin{array}{l}\text { Control } \\
\text { Intervención }\end{array}$ & 0,001 & 0,031 & 0,001 & 0,001 \\
\hline
\end{tabular}

Comparación de Significancia estadística de las pruebas de Wilcoxon de rangos con signo del grupo intervención y grupo control DNL. En la primera revaloración se evidenció que solo hubo significancia estadística en la dimensión de forma para el grupo control y de contenido para el grupo estimulado. En la segunda y tercera revaloración se evidenció significancia estadística en la dimensión de contenido en el grupo intervenido (afectando los totales) lo cual indica que, en este aspecto, las habilidades de estos niños mejoraron en comparación a la evaluación inicial. La tabla 2 muestra que en las otras dimensiones no se evidenció significancia estadística, debido a que estas mantuvieron su puntuación a lo largo de las revaloraciones aplicadas.

Tabla 2.

Comparación de Significancia estadística de las pruebas de Wilcoxon de rangos con signo del grupo intervención y grupo control DNL.

\section{Evaluaciones y revaloraciones}

Relación de la Primera Revaloración y Evaluación Inicial del lenguaje

Grupo Forma Contenido

Control

Intervención

$0,034 \quad 0,066$

0,010

Uso

0,317

0,063

Uso

1,000

1,000

0,705

0,027

0,157

del lenguaje

Contenido

Forma

0,317

0,655
0,480

0,026
Uso

1,000

1,000
Total

0,024

0,098

Total

0,317

0,016

Total

0,705

0,026

\section{Discusión}

La investigación reportó efectividad en el programa de estimulación temprana del lenguaje ejecutado, puesto que favoreció la adquisición y desarrollo lingüístico en niños con retraso simple del lenguaje. Al finalizar los 8 meses de estimulación, el grupo de intervención logró alcanzar el perfil de normalidad en la puntuación total de la prueba PLON-R en un $92 \%$ mientras que, los participantes del grupo control sólo alcanzaron el perfil de normalidad en un 14\%, es decir, 
los niños con retraso simple estimulados normalizaron sus habilidades lingüísticas en comparación al grupo control. De igual manera, sucedió con los niños sin retraso simple, los cuales potencializaron sus competencias comunicativas en promedios superiores a los no estimulados.

Estos resultados coincidieron con en el estudio realizado en Michoacán, México en el cual, se comprobó que antes de iniciar la "estimulación temprana" el desarrollo del grupo experimental era superior al del grupo control, pero la diferencia fue aún de mayor significancia al concluir el estudio (Pando, Aranda, Amezcua, Salazar \& Torres, 2004). Las explicaciones a estos hallazgos se soportan en las investigaciones de Del Rio (1987, citado en Brodal, 2010) y Luciana (2003) quienes afirman que, en las edades de 3, 6 y 11 años hay un gran despegue en el desarrollo del lenguaje y que en la etapa de los 3 años, la plasticidad del cerebro aún está en proceso, pero en niños con RSL deben estimularse áreas específicas del lenguaje para el desarrollo y fortalecimiento de sus habilidades lingüísticas. En este mismo sentido Sánchez y Villada (2014), también probaron la efectividad de los programas de estimulación del lenguaje, pero, sobre la capacidad de resolución de problemas en menores de 6 años escolarizados, ellos concluyeron que los niños que recibieron las sesiones de estimulación del lenguaje lograron solucionar situaciones problema en menos tiempo que los controles.

Con la ejecución del programa en la dimensión forma del lenguaje, se evidenció que en el grupo estimulado, el 92\% alcanzó normalidad mientras que, en el grupo control se encontró un $72 \%$ con este perfil. Lo que refleja que, aunque los niños estimulados normalizaron sus habilidades fonéticas-fonológicas con un mejor porcentaje, fue notorio que estos niveles tardan más tiempo en ser desarrollados por los patrones fonéticos que deben automatizar en el lenguaje repetido, inducido y espontáneo; esto se puede explicar teniendo en cuenta que en el retraso simple del lenguaje las falencias comunicativas son más evidentes en la expresión que en la comprensión y con la estimulación temprana se propicia el desarrollo de las áreas cerebrales, ya que se aprovecha su capacidad y plasticidad mejorando o potenciando sus funciones cognitivas y lingüísticas (Cerna, 2015).

El lenguaje, como función superior depende de la interacción unida de ciertas áreas cerebrales corticales y subcorticales (Diéguez \& Peña, 2012), dentro de estas para la dimensión forma se destaca el Área de Broca que participa en la formulación verbal (morfosintaxis) que corresponde a la expresión y comprensión de estructuras sintácticas, y cumple un rol en el procesamiento de verbos. Además, Participa activamente en la organización motriz del proceso articulatorio de la palabra hablada por su parte, el Área de Wernicke es responsable de la comprensión auditiva y el procesamiento léxico y la Circunvolución supramarginal se responsabiliza del procesamiento fonológico (González, 2011). Por ende, al direccionar adecuadamente la estimulación temprana del lenguaje se optimizan las funciones conjuntas de dichas áreas evidenciándose un mejor desempeño lingüístico en los grupos estimulados con y sin retraso simple del lenguaje.

En cuanto al aspecto semántico y pragmático, en la evaluación previa a la implementación del programa de estimulación los niños del grupo a intervenir con RSL, mostraron dificultades significativas en las dimensiones de contenido y uso, lo que se corrobora con los resultados encontrados por Petano (2009), en la cual se encontró que el 60\% de sus usuarios con retraso simple del lenguaje en edades de 4 años manifestaron problemas en el desarrollo pragmático y semántico, indicando que en un Retraso Simple estos aspectos del lenguaje se convierten en una constante que caracteriza la sintomatología de dicha patología comunicativa.

Por otra parte, después de realizadas las diferentes revaloraciones en los niños del grupo control con RSL es evidente que persisten las dificultades en el nivel semántico del lenguaje, lo cual puede explicarse según los postulados de Vigostky, Bruner y Garton que para la adquisición del lenguaje juegan un papel primordial el contexto y las variables socio-afectivas; desde esta perspectiva, el desarrollo semántico del niño depende de la calidad de las interacciones de este con su medio, de cómo utilizan el lenguaje los que le rodean, teniéndose en cuenta variables de tipos familiares, socio-económicas y educativas (Amar, Abello \& Tirado, 2004).

En cuanto a los niños del grupo de intervención con DNL fue notorio que mejoraron sus habilidades a nivel semántico y potencializaron sus habilidades en las otras dimensiones del lenguaje, lo cual no ocurrió en el grupo control. Según González (2007) esto se debe a que la estimulación temprana es un grupo de técnicas educativas especiales empleadas en niños entre el nacimiento y los seis años de vida, con el fin de posibilitar la adopción de medidas encaminadas a la 
prevención, la detección precoz de los riesgos de deficiencias y la intervención de los diversos trastornos del desarrollo, lo cual no sólo aplica para niños que tienen o están en riesgo de adquirir un retraso en el desarrollo, sino también para niños sanos, permitiendo estimular y potencializar sus habilidades.

En conclusión, el programa de estimulación diseñado y ejecutado fue efectivo, pues permitió que los niños estimulados mejoraran sus habilidades de lenguaje en contraste con lo no estimulados. Por lo tanto, se recomienda promover el diseño e implementación de este tipo de programas en los diferentes Hogares Infantiles y Centros de Desarrollo Infantil, para favorecer los niveles lingüísticos de la población que asiste a dichas instituciones.

\section{Conflicto de Intereses}

Los autores declaran no presentar ninguna relación de interés comercial o personal dentro del marco de la investigación que condujo a la producción del manuscrito.

\section{Colaboraciones}

Madelin María Palacio Vásquez: Gestión general de la investigación, trabajo de campo y redacción del artículo. Yaniris del Carmen Álvarez Pérez: Trabajo de campo y redacción del artículo.

Ana María Gómez Benítez: Trabajo de campo y redacción del artículo.

Marta Lucía Hernández Blanco: Apoyo a trabajo de campo y redacción del artículo.

Pedro José Blanco Tuirán: Asesor general.

Justo Rafael Fuentes Cuello: Asesor estadístico y metodológico 
Aguinada, G., Armentia, M.L., Fraile, A., Olangua, P. \& Uriz, N. (2005). PLON-R: Prueba de Lenguaje Oral de Navarra Revisada. Madrid: TEA Ediciones.

Alegre, J. \& Pérez, M. (2008). Guía práctica de los trastornos del lenguaje: descripción e intervención. Barcelona:Lebón.

Amar, J., Abello, R \& Tirado, D. (2004). Desarrollo infantil y Construcción del Mundo Social. Barranquilla, Colombia: Ediciones Uninorte, $152-180$

Attanasio, O., \& Gómez, M. (2012). Desarrollo infantil temprano y brecha socio-económica. Evidencia en niños y niñas de 6-42 meses, Bogotá, Colombia: División de salud y Protección Social, Banco Interamericano de Desarrollo.

Azcoaga, J. (1992). Las Funciones Cerebrales Superiores y sus alteraciones en el niño y en el adulto. Buenos Aires, Argentina: Paidós Ed.

Brodal, P. (2010). The central nervous system: structure and function. Oxford, Reino Unido: Oxford University Press

Cerna. V, C. (2015). La estimulación temprana en el desarrollo infantil de Los niños y niñas del primer ciclo de educación inicial. In Crescendo. Educación y Humanidades, 2, 184-19o. Recuperado de https://revistas.uladech. edu.pe/index.php/index/search/search?query=La+estimulaci $\% \mathrm{C}_{3} \% \mathrm{~B} 3 \mathrm{n}+$ temprana+en+el+desarrollo+infantil+de+Los+ni\% $\mathrm{C}_{3} \% \mathrm{~B} 10$ s $+\mathrm{y}+\mathrm{ni} \% \mathrm{C}_{3} \% \mathrm{~B} 1 \mathrm{as}+\mathrm{del}+$ primer+ciclo+de+educaci\% $\mathrm{C}_{3} \% \mathrm{~B} 3 \mathrm{n}+\mathrm{inicial} \&$ searchJournal=\&author$\mathrm{s}=\&$ title $=\&$ abstract $=\&$ galleyFull Text $=\&$ suppFiles $=\&$ dateFromMonth $=\&$ dateFromDay=\&dateFromYear $=\& d a t e T-$ oMonth $=\&$ date ToDay $=\&$ date ToYear $=\&$ date ToHour $=23 \&$ dateToMinute $=59 \&$ date ToSecond $=59 \&$ discipline $=\&$ sub ject $=\&$ type $=\&$ coverage $=\&$ indexTerms $=$

De la Torre, M., Guerrero, M., Conde, I \& Claros, R. (2002). Komunika: Programa para el desarrollo del conocimiento. Madrid, España: Aljibe Ed.

Diéguez, F \& Peña, J. (2012). Cerebro y lenguaje. Sintomatología Neurolingüística. Madrid, España: Editorial médica panamericana.

Equipo Facemamá. (2015). ¿Qué es la estimulación temprana? Facemamá.com recuperado de http://www.facemama.com/ estimulacion-temprana/ique-es-la-estimulacion-temprana.html

Gallardo, J. \& Gallego, J. (1995). Manual de logopedia escolar. Málaga, España: Aljibe.

González R. (2011). Trastorno del Lenguaje y Habla. En: Yáñez A. Neurología Fundamental. Santiago, Chile: Mediterráneo, $33-46$.

González, C. (2007). Los programas de Estimulación temprana desde la perspectiva del maestro. Liberabit, 13(13), 19-27. Recuperado de http://www.scielo.org.pe/scielo.php?script=sci_arttext\&pid=S1729-48272007000100003\&lng=es\&tlng=en.

González, O. (2013). Retraso simple del lenguaje. EcuRed.cu. Recuperado de http://www.ecured.cu/retraso_simple_del_lenguaje. Hernández, S., Fernández, F. \& Baptista, P. (2014). Metodología de la investigación. México D.F, México: Mc. Graw Hill.

Hernanz, M., \& Brucart, J. (1987). La sintaxis: Principios teóricos, la oración simple. Barcelona, España: Editorial Crítica.

INSOR. (2009). Instrumentos de tamizaje para la detección temprana de deficiencias auditivas en los menores de cinco años y escolares. Recuperado de http://www.insor.gov.co/historico/images/PUBLICACIONES/cartilla_tamizaje_menores_5_aos.pdf.

Lalangui, C., Romero, M., \& Escudero, L. (2014). La estimulación temprana y su incidencia en el desarrollo del lenguaje oral en las niñas y niños de los centros de educación inicial ciudad de Loja y Graciela Atarihuana de cueva año lectivo 2009 - 2010. (Tesis de pregrado). Recuperado de http://dspace.unl.edu.ec/jspui/handle/123456789/7170.

Luciana, M. (2003). Cognitive development in children born preterm: implications for theories of brain plasticity following early injury. Development and Psychopathology,15, 1017-1047. Recuperado de https://www.cambridge.org/core/ journals/development-and-psychopathology/article/cognitive-development-in-children-born-preterm-implications-for-theories-of-brain-plasticity-following-early-injury/A8F4BC2501565DC5617B485ED5698B77

Majnemer, A. (1998). Benefits of Early Intervention for Children with Developmental Disabilities. Seminars in Pediatric Neurology, 5, 62-69. Recuperado de https://www.sciencedirect.com/science/article/pii/S107190919880020X

Maldonado, M., \& Oliva, A. (2008). La estimulación temprana para el desarrollo afectivo, cognitivo y psicomotriz en niños y niñas síndrome de Down de o-6 años. (Tesis de pregrado). Universidad de san Carlos de Guatemala, Guate- 
mala. Recuperado de https://es.scribd.com/document/90916865/tesis

Miretti, M. (2003). La lengua oral en la Educación Inicial. San Diego, Estados Unidos de América: Homosapiens.

Pando, M., Aranda, B., Amezcua, S., Salazar, E., y Torres, L. (2004). Estimulación temprana en niños menores de 4 años de familias marginadas. Revista Mexicana de Pediatría, 71, 273-277. Recuperado de https://www.academia.edu/15229310/ Estimulaci\%C3\%B3n_temprana_en_ni\%C3\%B10s_menores_de_4_a\% 3 3\%B10s_de_familias_marginadas

Peña, J. (2014). Manual de Logopedia. Barcelona, España: Masson.

Petano, V. (2009). Atención Fonoaudiológica a los pacientes de la ESE Cartagena de India del Municipio de Corozal Sucre. (Propuesta de pasantía para obtener el título de fonoaudióloga). Universidad de Sucre. Recuperado de http://repositorio. unisucre.edu.co/bitstream/oo1/199/2/T616.85505\%20\%20P477.pdf, 11- 16 .

Reyes Cuervo, M., \& Bayona González, C. (2010). Desarrollo del lenguaje y vulnerabilidad: iprejuicio o fundamento científico?. Revista Colombiana De Rehabilitación, 10(1), 6-15. https://doi.org/10.30788/RevColReh.v10.n1.2011.72

Sánchez, J., \& Villada, J. (2014). Efectividad de un programa de estimulación del lenguaje sobre la capacidad de solución de problemas de niños de seis años escolarizados. Estudios Pedagógicos (Valdivia), 40(2), 361-372. Recuperado de https://dx.doi.org/10.4067/So718-07052014000300021

Saussure, F. (1973). Naturaleza del signo lingüístico. Habana. Cuba: Planeta-Agostini.

Secretaría de Salud de México. (s.f.). Vigilancia de la Nutrición y Crecimiento del Niño. Manual de capacitación para personal de salud. Recuperado de http://www.salud.gob.mx/unidades/cdi/documentos/5813.pdf

Serna, L. \& Gil, A. (2014). Programa de estimulación en alumnado con retraso simple del lenguaje. (Trabajo de grado). Universidad de Vallodolid, España. Recuperado de http://uvadoc.uva.es/handle/10324/

Team., R. (2015). A language and environment for statistical computing. Vienna, Austria: R Foundation for Statistical Computing. Recuperado de https://www.R-project.org/

\section{Notas}

El presente artículo se derivó de la investigación titulada "Influencia de un programa de estimulación temprana en el desarrollo lingüístico en niños de 3 años del municipio de Sincelejo-Sucre” financiada por la Universidad de Sucre y con Resolución 76 del 2014 del Consejo Académico.

Información de autores:

\section{Madelin María Palacio Vásquez}

Fonoaudióloga. MSc. en Patologías de Habla y Lenguaje

Universidad de Sucre

madelin.palacio@unisucre.edu.co

https://orcid.org/oooo-0oo3-426o-7540

\section{Yaniris del Carmen Álvarez Pérez}

Fonoaudióloga. MSc. en Neuropsicología y Educación

Universidad de Sucre

yanirisalvarezperez@gmail.com

\section{Ana María Gómez Benítez}

Fonoaudióloga.

Medicina integral IPS, Cartagena, Bolivar

anamariagomezbenitez@hotmail.com

\section{Marta Lucía Hernández Blanco}

Fonoaudióloga. MSc. en Discapacidad.

Universidad de Sucre

maluherblao1@gmail.com

\section{Pedro José Blanco Tuirán}

Médico. PhD. en Ciencias Básicas Biomédicas.

Universidad de Sucre

pedro.blanco@unisucre.edu.co

\section{Justo Rafael Fuentes Cuello}

Ingeniero Agrícola. Esp. en Gerencia de la

Educación y en Biometría.

Universidad de Sucre

justorfc@gmail.com 